\title{
Wave propagation in fractal-inspired self-similar beam lattices
}

\section{Citation}

Lim, Qi Jian, Pai Wang, Soo Jin Adrian Koh, Eng Huat Khoo, and Katia Bertoldi. "Wave propagation in fractal-inspired self-similar beam lattices." Applied Physics Letters 107, no. 22 (2015): 221911.

\section{Published Version}

doi:doi.org/10.1063/1.4936564

\section{Permanent link}

http://nrs.harvard.edu/urn-3:HUL.InstRepos:27657496

\section{Terms of Use}

This article was downloaded from Harvard University's DASH repository, and is made available under the terms and conditions applicable to Other Posted Material, as set forth at http:// nrs.harvard.edu/urn-3:HUL.InstRepos:dash.current.terms-of-use\#LAA

\section{Share Your Story}

The Harvard community has made this article openly available.

Please share how this access benefits you. Submit a story.

\section{Accessibility}




\title{
Wave propagation in fractal-inspired self-similar beam lattices
}

\author{
Qi Jian Lim, ${ }^{1}$ Pai Wang, ${ }^{2}$ Soo Jin Adrian Koh, ${ }^{1,3}$ Eng Huat Khoo, ${ }^{1,4}$ and Katia Bertoldi ${ }^{2,5}$ \\ ${ }^{1}$ Engineering Science Programme, National University of Singapore, Singapore 117576 \\ ${ }^{2}$ Harvard John A. Paulson School of Engineering and Applied Sciences, Harvard University, Cambridge, \\ Massachusetts 02138, USA \\ ${ }^{3}$ Department of Mechanical Engineering, National University of Singapore, Singapore 117576 \\ ${ }^{4} A^{*}$ STAR Institute of High Performance Computing, Singapore 138632 \\ ${ }^{5}$ Kavli Institute, Harvard University, Cambridge, Massachusetts 02138, USA
}

(Received 19 June 2015; accepted 13 November 2015; published online 3 December 2015)

\begin{abstract}
We combine numerical analysis and experiments to investigate the effect of hierarchy on the propagation of elastic waves in triangular beam lattices. While the response of the triangular lattice is characterized by a locally resonant band gap, both Bragg-type and locally resonant gaps are found for the hierarchical lattice. Therefore, our results demonstrate that structural hierarchy can be exploited to introduce an additional type of band gaps, providing a robust strategy for the design of lattice-based metamaterials with hybrid band gap properties (i.e., possessing band gaps that arises from both Bragg scattering and localized resonance). (C) 2015 AIP Publishing LLC.
\end{abstract}

[http://dx.doi.org/10.1063/1.4936564]

Phononic crystals ${ }^{1,2}$ and acoustic metamaterials ${ }^{3-7}$ have attracted significant attention in recent years ${ }^{8,9}$ both because of their rich physics and of their broad range of applications. These include wave guiding, ${ }^{10-12}$ frequency modulation, ${ }^{13,14}$ noise/vibration reduction, ${ }^{15,16}$ acoustic imaging, ${ }^{17-20}$ and thermal management. ${ }^{21,22}$ An important characteristic of these composite structures is their ability to manipulate the propagation of elastic waves through band gaps-frequency ranges of strong wave attenuation. In phononic crystals, band gaps are generated by Bragg-type scattering, whereas in acoustic metamaterials, localized resonances within the medium are exploited to attenuate the propagation of waves.

Materials with structural hierarchy are ubiquitous in natural and man-made systems ${ }^{23-26}$ and have recently received considerable interest because of their superior properties. ${ }^{27-32}$ It has also been shown that structural hierarchy can be exploited to manipulate the propagation of elastic waves. ${ }^{33-35}$ However, while all previous studies have focused on hierarchical phononic crystals, the effect of hierarchy on lattice-based acoustic metamaterials has not been explored yet.

In this letter, we focus on the dynamic response of fractal-like triangular beam lattices and investigate both numerically and experimentally the effect of hierarchy on the propagation of small amplitude elastic waves. While a simple triangular lattice is characterized by a locally resonant band gap, ${ }^{36}$ we find that fractal-like triangular lattices exhibit two types of gaps: (i) locally resonant band gaps and (ii) Bragg-type band gaps due to scattering. Locally resonant gaps are found in correspondence of the natural frequencies of the beams, whereas the stiffer regions introduced by the hierarchical refinement into the lattice are responsible for Bragg-type gaps. Our analysis reveals that, by introducing structural hierarchy into the lattice, not only higher frequency band gaps can be created, but also the mechanism responsible for such band gaps can be tuned.

To generate the hierarchical triangular lattice considered in this study, we start with an hexagonal unit cell comprising 24 equilateral triangles of edge $L$ (see Fig. 1(a)) and create
24 smaller triangles by connecting the edge centers of the 6 central triangles (see Fig. 1(b)). Clearly, this process can be repeated to create triangular lattices of higher hierarchical order and after $k$ iterations each original unit comprises 24 triangles of edge $L / 2^{k}$ and 18 triangles of edge $L / 2^{k-j}$ (with $j=1, \ldots, k)$. Therefore, a structure with $k$ orders of hierarchy comprises beams of slenderness $\lambda_{j}=L /\left(2^{j} b\right) \quad$ (with $j=0, \ldots, k)$, where $b$ denotes the width of the beam.

We start with a triangular lattice comprising beams of slenderness $\lambda_{0}=50$ and then introduce one order of hierarchy by adding beams of slenderness $\lambda_{1}=25$. All models are constructed using planar Euler-Bernoulli beams with width $b$, mass per unit length $m$, and made of a linear elastic isotropic material with Young's modulus $E$. We further assume that all joints are welded.

The Finite Element (FE) commercial package Abaqus/ Standard is used to investigate numerically the propagation of elastic waves both in infinite and finite-size lattices. The dynamic response of the infinite lattice is studied by considering a unit cell with Bloch-type boundary conditions and performing frequency-domain wave propagation analysis. ${ }^{7,11,37}$
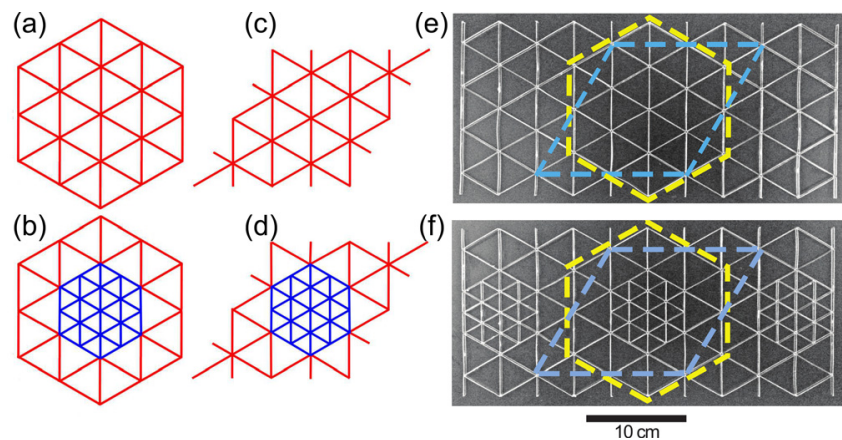

FIG. 1. (a) Hexagonal unit cell for the triangular lattice structure. (b) Hexagonal unit cell for the triangular lattice with one order of hierarchy. (c) Rhombic unit cell for the triangular lattice. (d) Rhombic unit cell for the triangular lattice with one order of hierarchy. (e) Acrylic sample of the triangular lattice. (f) Acrylic sample of the triangular lattice with one order of hierarchy. 
Note that we simplify the computational implementation by using rhombic unit cells in Figs. 1(c) and 1(d) instead of the hexagonal ones shown in Figs. 1(a) and 1(b). Moreover, steady-state analyses are conducted to calculate the transmission of finite-size lattices comprising different numbers of unit cells. In these simulations, an harmonic displacement is applied at the central node on the left edge of the model. The displacement of the central node on the right edge of the model is then monitored and the transmission is calculated as the ratio between the amplitudes of the output and input displacements (see supplementary material for more details ${ }^{38}$ ).

In addition to the numerical analysis, acrylic samples of the simple triangular lattice and the triangular lattice with one order of hierarchy are fabricated and tested. These samples are cut from a sheet of acrylic material of thickness $0.5 \mathrm{~cm}$ (with Young's modulus $E=2.8 \mathrm{GPa}$ and density $\rho=1190 \mathrm{~kg} / \mathrm{m}^{3}$ ) with a VLS6.60 laser cutter machine (equipped with a $60 \mathrm{~W} \mathrm{CO}_{2}$ laser). Each specimen comprises an array of $3 \times 1$ unit cells and measures $39.5 \mathrm{~cm} \times 20.5 \mathrm{~cm}$ (see Figs. 1(e) and 1(f)). Wave propagation in each sample is excited by an electrodynamic shaker (Brüel \& Kjaer-model LDS V406) attached to the left edge, and the dynamic response is recorded using miniature piezoelectric accelerometers (DJB Instruments-model A/25/E) attached at both ends of the sample. Finally, the transmittance is computed as the ratio between the output and input acceleration signals.

We start by investigating numerically the propagation of elastic waves in the triangular lattice. Fig. 2(a) shows the band structures in terms of the dimensionless frequency $\Omega=\omega / \omega_{\text {welded }}$, where $\omega_{\text {welded }}=22.4 \sqrt{E I /\left(m L^{4}\right)}$ is the first natural frequency of a single beam of length $L$ with both ends fixed (welded). As recently noticed, ${ }^{36}$ the structure is characterized by a band gap generated by local resonance. This finding is clearly supported by the fact that the band at the lower edge of the band gap is completely flat (see red band in Fig. 2(a)) and that is located in correspondence of the first natural frequency of the beams (i.e., $\Omega=1$ ). Furthermore, the Bloch mode shapes of the flat band at the high-symmetry points $\mathrm{G}, \mathrm{X}$, and M reported in Fig. 2(a) confirm that each beam vibrates independently according to its natural mode. A similar flat band can be observed at $\Omega=2.7$ in correspondence of the second natural frequency of the single beam. However, this second flat band does not give rise to a band gap, in agreement with previous studies. ${ }^{11,36}$

Next, we simulate the dynamic response of the triangular lattice with one order of hierarchy. The results reported in Fig. 2(b) clearly show that this system has a very different band dispersion behaviour. While the gap at $\Omega=1$ is retained, three additional band gaps appear at $\Omega=2.98-3.00, \Omega=3.13$ -3.35 , and $\Omega=3.43-3.87$. Importantly, all these three band gaps are located at frequencies far from the natural frequencies of elastic beams of length $L$ and $L / 2$ and the bands at their edges are not flat, suggesting they are not generated by local resonance, but by Bragg scattering (note that no locally resonant band gap is found at $\Omega=4$ - see supplementary material for more details). In particular, focusing on the band gap at $\Omega=3.43-3.87$, we can see that the bands at the lower (highlighted in purple) and upper (highlighted in orange) edges of the gap are not flat close to the $\mathrm{X}$ point (see Fig. 2(c)). Therefore, for certain wave vectors $\mathbf{k}$ the group velocity (a)

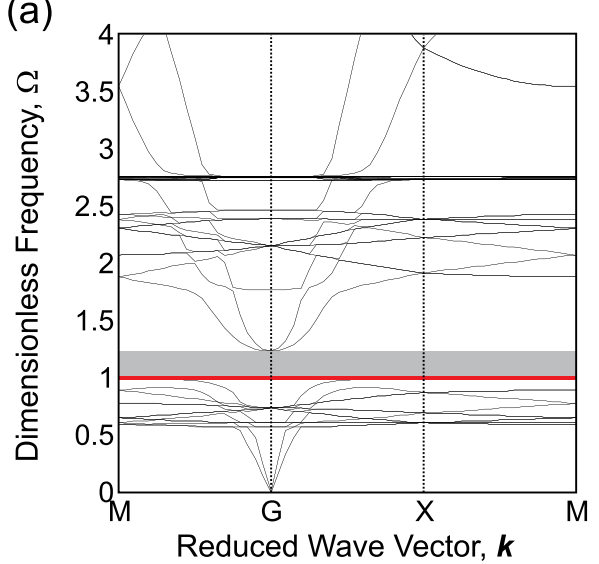

Red band

G

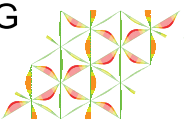

$X$

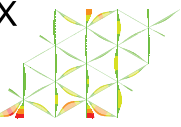

M

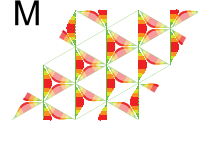

(b)

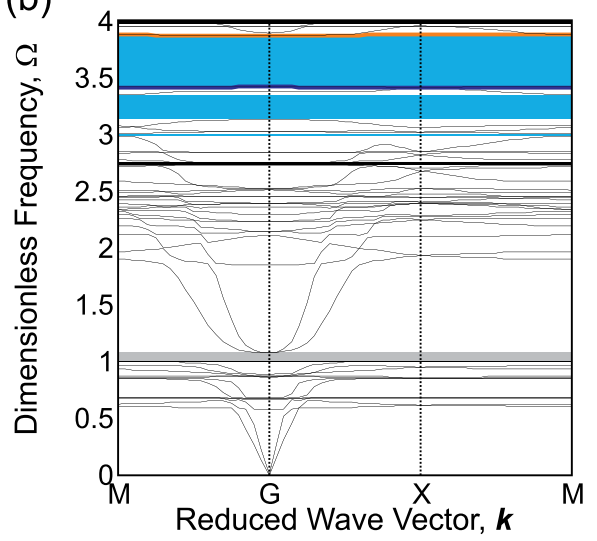

Purple band

G

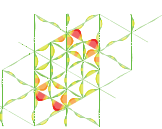

X

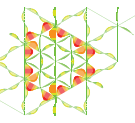

M
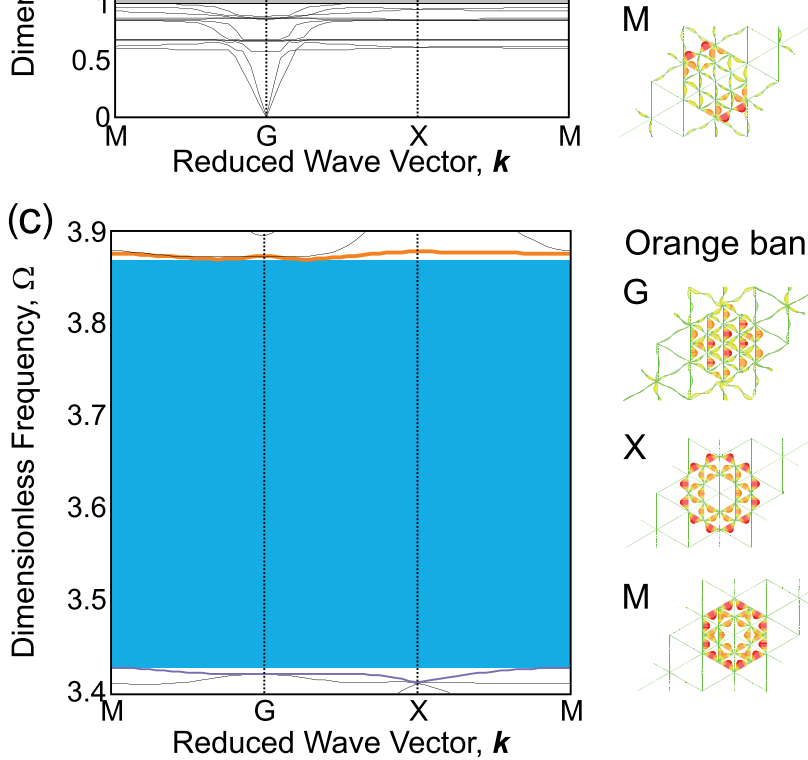

Orange band

G

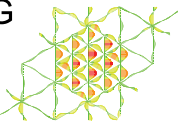

$X$

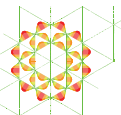

M

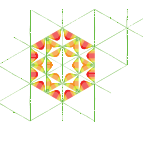

FIG. 2. (a) Dispersion relation of the triangular lattice. (b) Dispersion relation of the triangular lattice with one order of hierarchy. (c) Zoom-in of the dispersion relation of the triangular lattice with one order of hierarchy. The Bloch modes of the red, purple and orange bands at high-symmetry points of the Brillouin zone $(\mathrm{G}, \mathrm{X}$, and $\mathrm{M})$ are shown on the right.

of the propagating wave is not zero, resulting in not localised eigenmodes (as shown in Fig. 2, right). Finally, we note that these Bragg-type band gaps are generated because of the contrast of the effective properties introduced by the hierarchical refinement within the structure. In fact, since for a triangular lattice, the effective stiffness, $\bar{E}$, and density, $\bar{\rho}$, are given by ${ }^{39}$

$$
\bar{E}=1.15 \frac{b}{L} E=1.15 \frac{E}{\lambda}, \quad \bar{\rho}=2 \sqrt{3} \frac{b}{L} \rho=2 \sqrt{3} \frac{\rho}{\lambda} .
$$

It is easy to see that the shorter beams introduced into the lattice by the hierarchical refinement result in denser and stiffer cores within the unit cell. 
Having demonstrated that infinite hierarchical triangular lattices are characterized by higher frequency band gaps and that these gaps are generated by Bragg scattering and not local resonance as for the case of the triangular lattice, we now investigate how this affects the transmission of finite-size structures. First, we numerically investigate the dynamic response of models comprising $1 \times 20$ unit cells and apply periodic boundary conditions on their horizontal edges (to mimic the response of structures that are infinitely long in vertical direction-see Fig. S2). As shown in Fig. 3(c) for the triangular lattice, a significant asymmetric drop in the transmittance is observed between $\Omega=1$ and $\Omega=1.24$ with a pronounced minimum at $\Omega=1$. The fact that the lowest transmittance is observed in the vicinity of the lower edge of the band gap predicted by the dispersion relation further confirms that the band gap is generated by local resonance. ${ }^{3,9}$ On the other hand, for the triangular lattice with one order of hierarchy, we still see drops in the transmittance in correspondence of the band gaps predicted by the Bloch wave analysis at $\Omega=2.98-3.00, \Omega=3.13-3.35$, and $\Omega=3.43-3.87$, but these are more symmetric (see Fig. 3(d)). This is a characteristic of Bragg-type band gaps. ${ }^{8,9}$

While the results reported in Figs. 3(c) and 3(d) are for structures comprising 20 unit cells along the horizontal direction and infinitely long in vertical direction, in Figs. 3(e) and 3(f), we show the evolutions of the transmission for finite-size samples with $1 \times 3$ unit cells (in this case, all edges are free). Because of both size and boundary effects, the transmittance is different, but all important signatures are retained.

Finally, in Figs. 3(g) and 3(h), we report the experimentally measured transmittance for the same structures (the samples are shown in Figs. 1(e) and 1(f)). For both tested structures, we find a strong attenuation in transmission in the vicinity of the numerically predicted gaps. In particular, for the triangular lattice, we observe a drop of $\sim 20 \mathrm{~dB}$ near $\Omega=1.0$, which corresponds to a physical frequency of $f=606 \mathrm{~Hz}$ for this sample (see Fig. 3(g)). For the triangular lattice with one level of hierarchy, instead we see two regions of strong attenuations in transmission in the vicinity of $\Omega=$ 3.1 and $\Omega=3.5$, which correspond to $f=1879 \mathrm{~Hz}$ and $f=2121 \mathrm{~Hz}$, respectively (see Fig. 3(h)). The Bragg-type nature of these two gaps is further confirmed by the fact that their wavelength is about twice the unit cell size. In fact, from the homogenized properties defined in Eq. (1), the shear wave speed of the homogenized media can be estimated to be $c \sim$ $530 \mathrm{~m} / \mathrm{s}$, resulting in a band gap wavelength $\lambda=c / f \sim 25.2$ $\mathrm{cm}$ (since the band gaps frequency is $\sim 2100 \mathrm{~Hz}$ ), which about twice the unit cell size (see Fig. 1(f)).

In summary, we have studied both numerically and experimentally the propagation of small amplitude elastic waves in fractal-inspired beam lattices. First, our results indicate that the locally resonant band gap at $\Omega=1$ that characterizes the dynamic response of the triangular lattice is retained when introducing hierarchy in the structure. Interestingly, the position of this gap is fully predictable (it always occurs at $\Omega=1$ ), facilitating the design of systems that suit the engineering constraints. Second, we have seen that by adding hierarchy, more band gaps are formed. Most of these are generated by Bragg scattering, since the hierarchical refinement introduces a contrast in the effective properties within the unit cell.

While systems with multiple bandgaps in both lower and higher frequency intervals have been reported before, ${ }^{40-45}$ our results presented here indicate that hybrid band gap properties can also be achieved in an elastic material using a simple building block such as a straight elastic beam, without embedding additional resonators. In fact, in
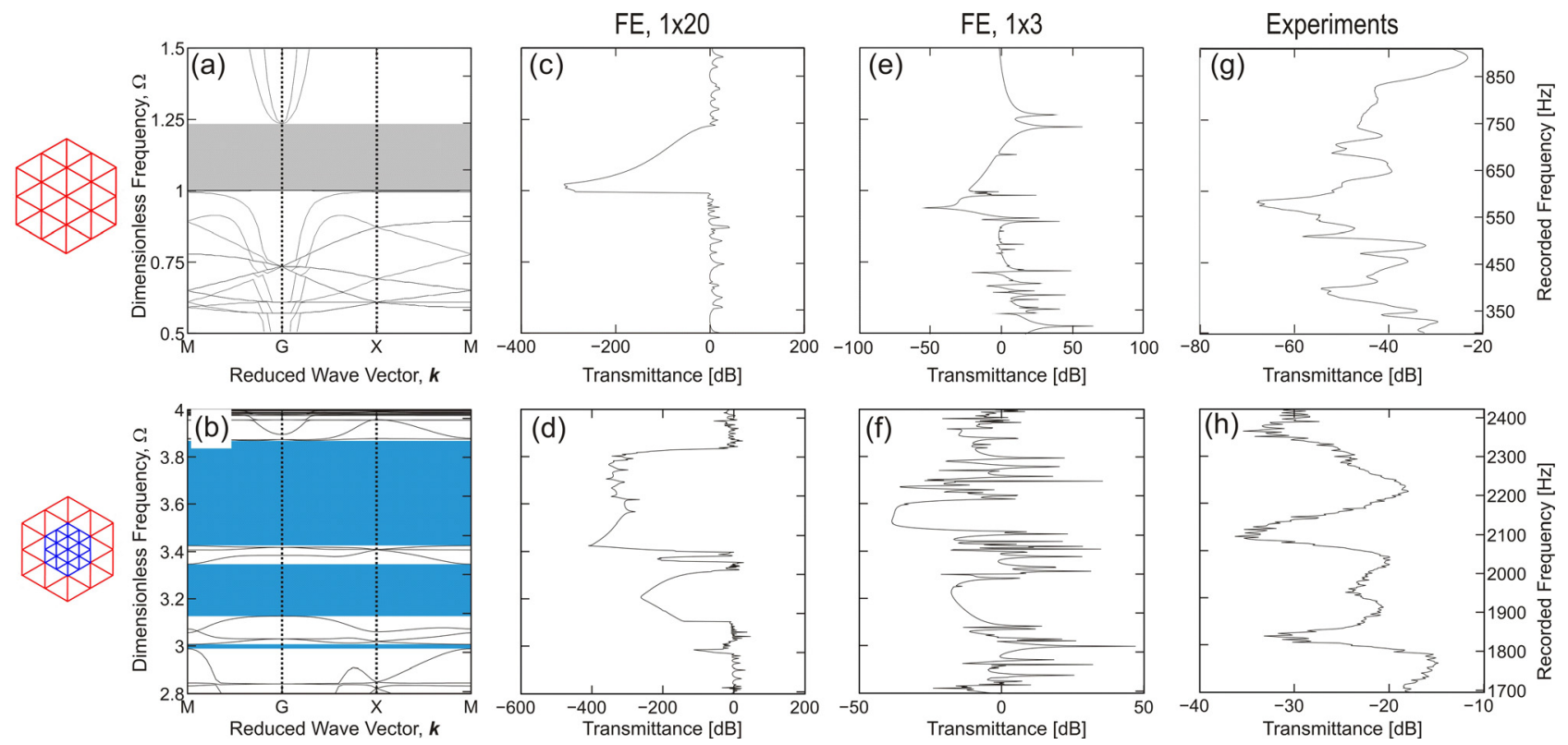

FIG. 3. (a) Dispersion plot of the triangular lattice. (b) Dispersion plot of the triangular lattice with one order of hierarchy. (c) Numerically calculated transmittance for the triangular lattice with $1 \times 20$ unit cells and periodic boundary conditions on the horizontal edges. (d) Numerically calculated transmittance for the triangular lattice with one order of hierarchy comprising $1 \times 20$ unit cells and with periodic boundary conditions on the horizontal edges. (e) Numerically calculated transmittance for the triangular lattice with $1 \times 3$ unit cells. (f) Numerically calculated transmittance for the triangular lattice with one order of hierarchy comprising $1 \times 3$ unit cell. $(\mathrm{g})$ Experimentally measured transmittance in a triangular lattice with $1 \times 3$ unit cells (see Fig. $1(\mathrm{e})$ ). (h) Experimentally measured transmittance in a triangular lattice with one order of hierarchy comprising $1 \times 3$ unit cells (see Fig. 1(f)). 
the proposed lattice-based metamaterials, the beams play simultaneously two roles: (i) they form a periodic elastic lattice with stiffer regions introduced because of the hierarchical refinement, so that Bragg-type band gaps are generated; (ii) they act themselves as mechanical resonators, resulting in the formation of locally resonant band gaps. Therefore, our results indicate a robust strategy to design acoustic metamaterials with hybrid band gap properties.

This work has been supported by Harvard MRSEC through Grant No. DMR-1420570 and by NSF through Grant Nos. CMMI-1120724 and CMMI-1149456 (CAREER). The authors would like to thank Bas Overvelde for help with illustrations.

${ }^{1}$ M. Sigalas and E. Economou, Solid State Commun. 86, 141 (1993).

${ }^{2}$ M. S. Kushwaha, P. Halevi, L. Dobrzynski, and B. Djafari-Rouhani, Phys. Rev. Lett. 71, 2022 (1993).

${ }^{3}$ Z. Liu, X. Zhang, Y. Mao, Y. Zhu, Z. Yang, C. Chan, and P. Sheng, Science 289, 1734 (2000).

${ }^{4}$ N. Fang, D. Xi, J. Xu, M. Ambati, W. Srituravanich, C. Sun, and X. Zhang, Nat. Mater. 5, 452 (2006).

${ }^{5}$ Y. Ding, Z. Liu, C. Qiu, and J. Shi, Phys. Rev. Lett. 99, 093904 (2007).

${ }^{6}$ Y. Lai, Y. Wu, P. Sheng, and Z. Zhang, Nat. Mater. 10, 620 (2011).

${ }^{7}$ P. Wang, F. Casadei, S. Shan, J. C. Weaver, and K. Bertoldi, Phys. Rev. Lett. 113, 014301 (2014).

${ }^{8}$ M. Maldovan and E. Thomas, Periodic Materials and Interference Lithography for Photonics, Phononics and Mechanics (Wiley-VCH, 2009).

${ }^{9}$ M. I. Hussein, M. J. Leamy, and M. Ruzzene, Appl. Mech. Rev. 66, 040802 (2014).

${ }^{10}$ M. Ruzzene, F. Scarpa, and F. Soranna, Smart Mater. Struct. 12, 363 (2003).

${ }^{11}$ A. S. Phani, J. Woodhouse, and N. A. Fleck, J. Acoust. Soc. Am. 119, 1995 (2006).

${ }^{12}$ J. O. Vasseur, A. Hennion, B. Rouhani, F. Duval, B. Dubus, and Y. Pennec, J. Appl. Phys. 101, 114904 (2007).

${ }^{13}$ M. Kafesaki, M. M. Sigalas, and N. Garcia, Phys. Rev. Lett. 85, 4044 (2000).

${ }^{14}$ Y. Pennec, B. Djafari-Rouhani, J. O. Vasseur, A. Khelif, and P. A. Deymier, Phys. Rev. E 69, 046608 (2004).

${ }^{15}$ J. Mei, G. Ma, M. Yang, Z. Yang, W. Wen, and P. Sheng, Nat. Commun. 3, 756 (2012)
${ }^{16}$ S. Brûlé, E. H. Javelaud, S. Enoch, and S. Guenneau, Phys. Rev. Lett. 112, 133901 (2014).

${ }^{17}$ L.-S. Chen, C.-H. Kuo, and Z. Ye, Appl. Phys. Lett. 85, 1072 (2004).

${ }^{18}$ J. Christensen, A. I. Fernandez-Dominguez, F. de Leon-Perez, L. MartinMoreno, and F. J. Garcia-Vidal, Nat. Phys. 3, 851 (2007).

${ }^{19}$ J. Li, L. Fok, X. Yin, G. Bartal, and X. Zhang, Nat. Mater. 8, 931 (2009).

${ }^{20}$ D. Bigoni, S. Guenneau, A. B. Movchan, and M. Brun, Phys. Rev. B 87, 174303 (2013).

${ }^{21}$ M. Maldovan, Phys. Rev. Lett. 110, 025902 (2013).

${ }^{22}$ B. L. Davis and M. I. Hussein, Phys. Rev. Lett. 112, 055505 (2014).

${ }^{23}$ B. Mandelbrot, The Fractal Geometry of Nature (W.H. Freemen and Company, New York, 1983).

${ }^{24}$ R. Lakes, Nature 361, 511 (1993).

${ }^{25}$ P. Fratzl and R. Weinkamer, Prog. Mater. Sci. 52, 1263 (2007).

${ }^{26}$ N. Pan, Appl. Phys. Rev. 1, 021302 (2014).

${ }^{27}$ A. Ajdari, B. H. Jahromi, J. Papadopoulos, H. Nayeb-Hashemi, and A. Vaziri, Int. J. Solids Struct. 49, 1413 (2012).

${ }^{28}$ R. Oftadeh, B. Haghpanah, D. Vella, A. Boudaoud, and A. Vaziri, Phys. Rev. Lett. 113, 104301 (2014).

${ }^{29}$ Z. Zhang, Y.-W. Zhang, and H. Gao, Proc. R. Soc. London, Ser. B 278, 519 (2011).

${ }^{30}$ F. Song, J. Zhou, X. Xu, Y. Xu, and Y. Bai, Phys. Rev. Lett. 100, 245502 (2008).

${ }^{31}$ Y. Li, Z.-Y. Fu, and B.-L. Su, Adv. Funct. Mater. 22, 4634 (2012).

${ }^{32}$ R. S. Lakes, J. Compos. Mater. 36, 287 (2002).

${ }^{33}$ Y. Xu, X. Tian, and C. Chen, Phys. B 407, 1995 (2012).

${ }^{34}$ Y. Xu, C. Chen, and X. Tian, J. Vib. Acoust. 136, 011011 (2013).

${ }^{35}$ D. Mousanezhad, S. Babaee, R. Ghosh, E. Mahdi, K. Bertoldi, and A. Vaziri, Phys. Rev. B 92, 104304 (2015).

${ }^{36}$ P. Wang, F. Casadei, S. H. Kang, and K. Bertoldi, Phys. Rev. B 91, 020103 (2015).

${ }^{37}$ P. Wang, J. Shim, and K. Bertoldi, Phys. Rev. B 88, 014304 (2013).

${ }^{38}$ See supplementary material at http://dx.doi.org/10.1063/1.4936564 for additional details, which includes the reference. ${ }^{8,36,37}$

${ }^{39}$ L. J. Gibson and M. F. Ashby, Cellular Solids: Structure and Properties (Cambridge University Press, 1997).

${ }^{40}$ L. Liu and M. I. Hussein, J. Appl. Mech. 79, 011003 (2011).

${ }^{41}$ Y. Achaoui, A. Khelif, S. Benchabane, L. Robert, and V. Laude, Phys. Rev. B 83, 104201 (2011).

${ }^{42}$ Y. Xu, C. Chen, and X. Tian, Chin. Phys. Lett. 30, 044301 (2013).

${ }^{43}$ Y.-F. Wang, Y.-S. Wang, and C. Zhang, J. Phys. D: Appl. Phys. 47, 485102 (2014).

${ }^{44}$ Y.-F. Wang, Y.-S. Wang, and C. Zhang, AIP Adv. 4, 124403 (2014).

${ }^{45}$ B. Sharma and C.-T. Sun, "Local resonance and Bragg bandgaps in sandwich beams containing periodically inserted resonators," preprint arXiv:1503.09048 (2015). 